\section{LAS PRIMERAS INSTITUCIONES CIENTÍFICAS ALEMANAS EN ESPAÑA: LOS COMIENZOS DE LA COOPERACIÓN INSTITUCIONAL EN LOS ALBORES DEL SIGLO XX*}

\author{
Sandra Rebok \\ Consejo Superior de Investigaciones Científicas \\ srebok@orgc.csic.es
}

\begin{abstract}
This article describes different German initiatives to institutionalize the scientific exchange with Spain during the interwar period. From the beginning these efforts were focused on the cities of Barcelona and Madrid and started up in 1923 with the inauguration in Barcelona of the Centro de Estudios Alemanes y de Intercambio, followed two years later by the Centro de Intercambio Intelectual Germano-Español. Both institutions were devoted to fomenting interest in German language and culture present in Spain in order to strengthen Spanish-German relationships in all contexts, including the administrative management of academic exchange. In addition, another relevant initiative would enter the scene in 1927: the Görres Institut in Madrid, motivated more by its Catholic roots and its dedication to research itself than to the exchange of information or scientists. In this way, along with other scientific institutions in the following years, a close scientific collaboration between both countries was made possible until the Spanish Civil War and the subsequent World War put an end to promising endeavors.
\end{abstract}

KEY WORDS: History of science, scientific cooperation, institutionalization of scientific exchange, Germany, Spain.

Como se ha venido recordando en numerosas ocasiones durante este año conmemorativo, en la España de comienzos del siglo XX la internacionalización de la ciencia en general, y el contacto científico-académico con Alemania en particular, se debe en primer término a la labor desempeñada por la Junta para la Ampliación de Estudios

\section{THE VERY FIRST GERMAN SCIENTIFIC INSTITUTIONS IN SPAIN. THE BEGINNING OF THE INSTITUTIONAL COOPERATION IN THE EARLY 20TH CENTURY}

RESUMEN: Este artículo describe distintas iniciativas alemanas para dar una forma institucional al intercambio científico con España en época de entreguerras. Estos esfuerzos se centraron desde un principio en las ciudades de Madrid y Barcelona y se iniciaron en el año 1923 con la inauguración del Centro de Estudios Alemanes y de Intercambio en Barcelona y continuaron dos años más tarde también en Madrid con la creación del Centro de Intercambio Intelectual Germano-Español. Ambas instituciones estaban dedicadas a reforzar el interés que había en España por la cultura y la lengua alemanas, a fomentar las relaciones culturales hispano-alemanas en todos los ámbitos, así como a la gestión administrativa del intercambio académico. A esto se unió en el año 1927 otra iniciativa de relevancia, si bien con una motivación de origen católico y dedicada más a la propia investigación que al intercambio de personas o de información: el madrileño Instituto Görres. Junto con otras instituciones científicas en los siguientes años se pudo fomentar una estrecha colaboración científica entre los dos paises, hasta que la Guerra Civil y la subsiguiente guerra mundial acabaron con muchos de sus prometedores proyectos.

PALABRAS CLAVE: Historia de la ciencia, cooperación científica, institucionalización del intercambio científico, Alemania, España.

(JAE) por medio de diversas iniciativas'. Al mismo tiempo surgía, por parte alemana, una serie de instituciones de diversa índole, cuyo propósito consistía en el intercambio científico con España. A continuación expondremos las, a nuestro juicio, más importantes de las instituciones alemanas creadas durante esta época. A fin de comprender 
mejor el contexto histórico-político en el que nacieron, y mostrar con mayor claridad cuál era el interés de Alemania por España en este sentido, nos referiremos brevemente en primer lugar a la difícil situación por la que atravesaba la ciencia alemana tras la Primera Guerra Mundial: ya durante la conflagración, se procedió por iniciativa francesa a la disolución de la Asociación Internacional de Academias, fundada en 1899. En las organizaciones científicas internacionales creadas posteriormente, Conseil International de Recherches (Ciencias Naturales) y Union Académique Internationale (Humanidades), no tuvieron cabida los científicos alemanes ${ }^{2}$. A efectos prácticos esto significaba la exclusión de Alemania de la cooperación cientifica internacional. Ante tales hechos, Fritz Haber y Friedrich Schmidt-Ott impulsaron la fundación de la Notgemeinschaft der deutschen Wissenschaft el 30 de octubre de 1920, que asumió durante el boicot instigado por las potencias de la Entente la tarea tanto de respaldar económicamente la ciencia doméstica como de servir de correa de transmisión entre la ciencia alemana y el extranjero ${ }^{3}$.

Por esta razón, en aquellos tiempos problemáticos Alemania se mostraba naturalmente agradecida ante cualquier gesto de buena voluntad por parte de otros países. Debido a la neutralidad política española durante la Gran Guerra y la escasa influencia que, por ende, tuvieron en este país las decisiones de los aliados, España adquirió una especial relevancia en el contexto de la posguerra. En ese intento de establecer posibles conexiones con la comunidad científica internacional, Alemania recordó las relaciones históricas con España. El agradecimiento y la confianza hacia España se vieron notablemente incrementados a raíz de un Ilamamiento dirigido por España a la conferencia de paz reunida en Versalles contra el boicot de la ciencia alemana, proclama que despertó la atención a escala internacional. El 28 de mayo de 1919 un grupo formado por 110 investigadores españoles, encabezado por Emilio Huguet del Villar, geógrafo de la Universidad de Madrid, elevó una protesta contra la exclusión de los investigadores alemanes de las organizaciones científicas internacionales, recordando en este sentido la valiosísima contribución de Alemania al avance científico. El Ilamamiento que suscribieron los científicos españoles concluía con las palabras:

"Ningún pueblo, y menos aún los que, como el alemán, y otros de la Europa Central, han contribuido tan brillantemente al progreso científico, quede en condiciones que dificulten en ellos el ulterior desarrollo de las ciencias y su legítima y benéfica influencia intelectual en el exterior"4.

Se hace aquí ostensible una tradición que en España se remonta a mucho tiempo atrás: junto los intelectuales, políticos, etc., que se orientaban hacia Alemania en múltiples aspectos, los Ilamados germanófilos, existía también el grupo de los francófilos, orientados más bien hacia el modelo cultural francés. Esta división se puso también de manifiesto tras la Primera Guerra Mundial: existía por un lado un grupo de científicos que mostraba cierta simpatía por Alemania en general -si bien no necesariamente por su política, sí por la cultura y la ciencia alemanas-, pero también se encontraban aquéllos que más bien se identificaban con los aliados. Por otro lado, Alemania siempre había visto especialmente a Francia como rival, aunque también como modelo a imagen del cual orientar las propias iniciativas, a la vista del éxito obtenido por las medidas adoptadas por el país galo en el ámbito de la política cultural. A diferencia de su país vecino, Alemania no había contado con una planificación de su política cultural exterior con anterioridad a la Primera Guerra Mundial. Había únicamente iniciativas particulares, como, por ejemplo, un intercambio con profesores estadounidenses, la creación de unos cuantos institutos de investigación en otros países, el sistema educativo extranjero, así como el fomento esporádico de viajes internacionales de estudios o a congresos. El intercambio docente entre Estados Unidos y Alemania se inició en el año $1905^{5}$ a instancias del emperador Guillermo II, siendo el primer proyecto de Alemania en materia de política cultural; su origen se debió a una iniciativa de Friedrich Althoff, Director de Universidades dentro del Ministerio de Cultura de Prusia (1839-1908) y fue, por ende, un primer intento de resaltar también en el exterior la importancia de Alemania para la ciencia e influir así de forma consciente en la imagen internacional de este país.

Tras el colapso político y militar de Alemania, existía más que nunca la obligación de transmitir valores espirituales y culturales para así llevar a cabo una sopesada política cultural alemana cara al exterior. Ya a comienzos de 1917 el Ministerio Prusiano de Cultura presentó una memoria, preparada por el historiador Paul Fridolin Kehr ${ }^{6}$, que contenía el esbozo de un plan para la divulgación de la cultura alemana en el exterior y que colocaba a España entre sus objetivos. En este orden de cosas ya se trató entonces la creación de un instituto español $\mathrm{l}^{7}$ y se dieron los primeros 
pasos para el fortalecimiento de las relaciones científicoacadémicas entre ambos paises en los años veinte del pasado siglo $\mathrm{XX}^{8}$.

Lo que además puede haber contribuido a forjar esta mirada alemana hacia España fueron las diversas actividades iniciadas y organizadas por la JAE, que también en Alemania fueron observadas y comentadas con gran interés en las distintas publicaciones. Sobre todo, revistas como Spanien. Zeitschrift für Auslandskunde, Mitteilungen aus Spanien e lberica informaban con regularidad sobre los distintos cursos ofrecidos por los centros de la JAE. Asimismo, el programa de pensiones de la JAE fue alabado y presentado como modelo a seguir, tal como muestra una publicación sobre los estudiantes en una comparación internacional:

"Los pensionados mantienen el contacto con la organización tanto durante su estancia en el extranjero como a su término $y$, al volver de su viaje, presentan un informe sobre su campo de trabajo y sus experiencias (...) Tiene especial importancia que la Junta provea de nuevos medios a los estudiantes que han vuelto del extranjero para que puedan aplicar sus estudios tras el título obtenido (...)"9.

\section{El Centro de Estudios Alemanes y de Intercambio de Barcelona y el Centro de INTERCambio Intelectual Germano-Español de Madrid}

La idea de establecer una sede para el intercambio científico hispano-alemán se concentró desde un principio en las ciudades de Madrid y Barcelona. No obstante, y a pesar de poseer una orientación conceptual análoga, tenía orígenes diversos.

El Centro de Estudios Alemanes y de Intercambio (Deutsche Wissenschaftliche Vermittlungsstelle) nació en Barcelona en el año 1923 a instancias del Cónsul General Ulrich von Hassell, por entonces representante diplomático de la República de Weimar ${ }^{10}$. El propósito que se perseguía era el de reforzar el interés que había en España por la cultura y la lengua alemanas. Por otra parte, von Hassell quería contrarrestar de manera explícita la hegemonía cultural francesa en España, surgida a raíz de una ofensiva impuesta por la política cultural del país galo. Después de diversos preparativos Ilevados a cabo desde el año 1922 pudo inaugurarse el Centro de Estudios el día 1 de marzo de 1923 en una oficina cedida por una empresa alemana en la Bajada San Miguel número 1 y bajo la dirección subsidiaria de Otto Schmidtborn, maestro del Instituto alemán de Enseñanza Media de Barcelona. La junta directiva estaba compuesta por el Cónsul General de Alemania y otros ciudadanos alemanes residentes en Barcelona, así como unos cuantos investigadores españoles ${ }^{11}$. Según el esbozo de su programa, su objetivo era el fomento de las "relaciones y del intercambio cultural hispano-alemán en todos los ámbitos"12; esta institución habría de hacerse cargo en primer término de la gestión administrativa que requiere el intercambio científico, pero no ser realmente un centro de investigación, como parece sugerir, al menos en parte, su denominación española. Como sea que el Centro disponía de personal limitado, sus tareas consistían fundamentalmente en proporcionar información, facilitar contactos entre científicos alemanes y españoles y organizar conferencias académicas, así como posibilitar el intercambio bibliográfico.

El Centro no recibió subvención estatal hasta el año 1925, financiándose exclusivamente a través de los donativos de su junta directiva. Por tal razón sus posibilidades eran más bien modestas y sólo se sabía de su existencia en un reducido círculo. En diciembre de 1928 cambiaron las tornas tras la mudanza al edifico anexo al Colegio Alemán y más aún a partir de finales de 1929, a consecuencia de la contratación de Karl Supprian como director, cuyo sueldo dependía ahora de los fondos destinados a cultura por el Ministerio de Asuntos Exteriores. En septiembre de 1931 el Centro de Estudios pudo al fin instalarse en sus propios locales del centro de Barcelona en la calle Moya 4. A partir de entonces se iniciaría la colaboración con diversas organizaciones científicas españolas, sobre todo con el Institut d'Estudis Catalans, la Biblioteca de Catalunya y el Ateneo Barcelonés ${ }^{13}$.

Bastante mayor calado y resonancia pública tuvo la labor desarrollada por el Centro de Intercambio Intelectual Germano-Español (Arbeitsstelle für deutsch-spanische Wissenschaftsbeziehungen), fundado en 1924 en Madrid. Como titular se consideró en primer lugar a la KaiserWilhelm-Gesellschaft (KWG), fundada en el año 1911, que contaba ya con vínculos con España $y$, al término de la Primera Guerra Mundial, se propuso investigar a conciencia la historia de este país. La iniciativa había partido del historiador Paul Fridolin Kehr (1860-1944), por entonces 
Director del Instituto de Historia de la KWG, quien llevaba realizando viajes de investigación a España desde el año 1922. La KWG se interesaba sobre todo por Carlos V y Felipe II, y llegó incluso a constituirse una pequeña comisión de trabajo que, sin embargo, no llegó a cuajar en la práctica ${ }^{14}$. Además de las iniciativas de esta sociedad, en 1917 hubo otras propuestas provenientes del círculo reunido en torno al Instituto Iberoamericano de Hamburgo, fundado ese mismo año, así como de la Asociación España-Alemania, con la que se comprometió muy especialmente el filólogo Bernhard Schädel (1878-1926). Después de ciertas dificultades a la hora de aunar voluntades se descartó la idea de afrontar una iniciativa común, de modo que la sección de cultura del Ministerio de Asuntos Exteriores siguió su propia hoja de ruta. Después de 1923 fue Georg Schreiber ${ }^{15}$, diputado del Centro y experto en Historia de la Iglesia, quien llevó adelante este nuevo planteamiento que desembocó finalmente en la inauguración oficial en Madrid de Centro en enero de 1925.

En este caso, los objetivos también se formularon en función de los éxitos cosechados por la política cultural gala, ya que se pretendía "asegurarse un sitio en España en el ámbito de la ciencia", para así "contrarrestar la propaganda cultural francesa"16. Otro de los intereses que tenía Alemania, y que se menciona siempre en los documentos relacionados con la fundación y la actividad de esta institución, era la dimensión de España como puente hacia el mundo iberoamericano:

"El hecho de que el Centro de Intercambio concibe su labor mediadora no en el reducido ámbito español, sino extendiéndolo también a lberoamérica, orienta su actividad no tanto hacia el fomento de las relaciones científicas con los círculos madrileños y las universidades de provincia, sino también hacia la toma de contacto con lberoamérica"17.

Por un lado, se buscaba otra salida más al aislamiento científico en que estaba sumida Alemania, pero por otro se perseguian también intereses de tinte claramente económico.

El Centro de Intercambio se radicó en un primer momento en el Instituto Alemán de Enseñanza Media situado en el número 15 de la calle Fortuny ${ }^{18}$, pero a partir de septiembre de 1927 continuó su actividad en un edificio propio, situado en un inmueble representativo de la calle Zurbano número 32. Fue al principio un servicio de atención a estudiantes o científicos españoles, que querían establecer contactos con Alemania o preparaban una estancia en Alemania. Durante mucho tiempo se dudó acerca de quién habría de asumir la dirección del Centro. Siguiendo la recomendación de Hugo Obermeier, paleontólogo alemán afincado en Madrid, la decisión recayó finalmente en Gerhard Moldenhauer ${ }^{19}$, un joven romanista que sería relevado, en diciembre de 1929, por Alfons Adams.

Según el primer informe de actividades del Centro de Intercambio de Madrid, que data de mayo de 1926, la idea central era que arte y ciencia, como "únicas fuerzas altruistas que unen los pueblos", están llamadas a "actuar como contrapeso frente a los enfrentamientos que generan los intereses políticos y comerciales de las naciones". En 1929 Moldenhauer resumía en los siguientes términos las tareas concretas del Centro de Intercambio:

"1. Informar por escrito o verbalmente sobre aspectos científicos a investigadores alemanes y españoles.

2. Impartir cursos de lengua y gestionar el intercambio lingüístico.

3. Organizar conferencias de eruditos españoles y alemanes.

4. Crear una biblioteca científica de acceso libre, con registro e intercambio de libros y publicaciones" 20 .

Como pone de manifiesto el director del Centro de Intercambio en su informe, en la práctica cotidiana la mayor parte del trabajo consistía entonces en el servicio de información y mediación: ahí tenía cabida la redacción de cartas de recomendación para españoles que marchaban a Alemania a estudiar (entre los que se encontraban aquéllos que lo hacían a través de la JAE), consejos para viajes y estudios, información para alemanes residentes en España, información sobre instituciones científicas en ambos países, así como asesoramiento y ayuda en los trámites de matrícula en las universidades. Además, se facilitaba el acceso a bibliografía especializada mediante la consulta detallada en archivos, bibliotecas y museos, se proporcionaban copias y se procuraban libros raros o descatalogados. Este servicio de información fue aglutinando de forma paulatina en torno al Centro de Intercambio a todas las instituciones y organismos públicos relacionados con España o Alemania, razón por la que experimentó un rápido aumento en el número de visitantes así como en el de consultas por escrito ${ }^{21}$. 
Importante tarea de esta institución la constituian también los cursos de idiomas: cuatro veces al año se ofrecian clases de español para alemanes; estos cursos fueron dirigidos por José Gavira y Martín, y estaban ante todo orientados a principiantes, constando de unas 40 a 60 horas lectivas. Además, tuvieron relevancia los ciclos de conferencias organizados por el Centro de Intercambio de Madrid, al menos dos veces al mes. Es de destacar que la selección de los ponentes se realizara según criterios estrictamente científicos, sin tener en cuenta las posibles convicciones políticas o religiosas ${ }^{22}$. Ya en los primeros momentos de la actividad del Centro, se contó entre los ponentes con científicos españoles tan destacados como José Rodríguez Carracido, Sebastián Recanséns, Rafael Altamira, Miguel Artigas, Pedro Bosch y Gimpera, José María Torroja, Blas Cabrera y Felipe, José Casares Gil y Enrique Moles. Entre los eruditos alemanes residentes en España o bien de paso en nuestro país, disertaron sobre sus temas de investigación, entre otros: Beda Kleinschmidt, Georg Weise, Karl Voretzsch, Gertrud Richert, Hugo Obermeier, H. Kluge, A. Hase, Hugo Kehrer y Richard Koch ${ }^{23}$. Estos investigadores procedian de diversas especialidades, sobre todo de la Medicina, la Filosofía, el Derecho y las ciencias aplicadas, proviniendo de los rincones más diversos de Alemania, Austria y Suiza, y testimoniando así el polifacético intercambio científico existente.

A fin de que las conferencias celebradas pudieran llegar a un círculo más amplio de interesados fuera de la capital de España, se publicó una selección de las mismas en la colección Conferencias dadas en el Centro de Intercambio Intelectual Germano-Español. Otra publicación del Centro de Intercambio fue el Boletín bibliográfico, de periodicidad cuatrimestral, dirigido por José Gavira y Martín, Gerhard Moldenhauer y Edmund Schramm. En él aparecieron reseñas de libros de especialistas españoles y alemanes para ofrecer una visión general e información sobre las novedades publicadas en todos los ámbitos. Además, a partir de 1927 se publicó la revista Investigación y progreso bajo la dirección de Hugo Obermaier y Antonio de Zulueta. La revista se fundó con miras al intercambio científico entre España y Alemania -siguiendo el ejemplo de la versión alemana Forschung und Fortschritt- y publicó los trabajos de investigadores de ambas naciones. Por otra parte, la edición de dichas publicaciones posibilitó al Centro de Intercambio la obtención, en tiempos de crisis, de otros títulos interesantes para su biblioteca mediante el inter- cambio, y gracias al apoyo de la Notgemeinschaft der deutschen Wissenschaft.

Grosso modo podemos afirmar que el Centro de Intercambio de Madrid consiguió, a pesar de contar con escaso personal y un presupuesto más bien modesto, una repercusión cada vez mayor. Consensuaba siempre su programa con la Embajada alemana y tenía múltiples contactos, personales e institucionales, en el ámbito de las relaciones científicas hispano-alemanas. También hubo numerosos puntos de contacto con la JAE, así como posibilidades de cooperación, por ejemplo a la hora de preparar a los becarios para su futura estancia en Alemania. Además, el Centro de Intercambio asumió una función mediadora entre la JAE y la Notgemeinschaft der deutschen Wissenschaft en el intercambio de publicaciones, así como en el nombramiento de lectores tanto para instituciones alemanas como españolas.

La posterior pervivencia del Centro de Intercambio de Madrid y del Centro de Estudios de Barcelona a partir de abril de 1934 quedó ligada al Deutscher Akademischer Austauschdienst (DAAD), fundado el 1 de enero de 1931, ya que ambas instituciones fueron nombradas como sus filiales en el extranjero, con lo que perdieron la autonomía de la que habían gozado hasta entonces, así como su orientación exclusivamente científica. Por su parte, el DAAD aglutinó la labor de tres organizaciones que le precedieron, y que habian surgido por diversas razones: el Akademischer Austauschdienst (AAD), fundado oficialmente en Heidelberg ${ }^{24}$ el 1 de enero de 1925, y que se desarrolló a raíz de una iniciativa ${ }^{25}$, surgida ya en 1922 , de Carl Joachim Friedrich, estudiante de Ciencias Sociales y Políticas; la Alexander von Humboldt-Stiftung (AvHSt), instaurada en 1925 por el Ministerio de Asuntos Exteriores y concebida desde un principio como instrumento de la política cultural del Estado en el exterior; y la Deutsche Akademische Auslandsstelle des Verbandes der Deutschen Hochschulen, que echó a andar en 1927 en Dresde ${ }^{26}$. El motivo principal de la fusión fue el reconocimiento de la necesidad de coordinar el trabajo académico en el extranjero en sus diversas facetas con el objetivo de movilizar la ciencia alemana como activo de la política cultural exterior y tener en cuenta la magnitud internacional de la ciencia. Las áreas de actuación más significativas del DAAD a lo largo del año 1931 fueron: el fomento del intercambio académico, la concesión de becas, la asistencia a estu- 
diantes extranjeros en Alemania, la información sobre los estudios en el extranjero, así como la publicación de la revista Hochschule und Ausland ${ }^{27}$.

Tras el ascenso de los nacionalsocialistas al poder, el DAAD -al igual que otras instituciones de corte científico o cultural- pasó a formar parte de los intereses del Tercer Reich en materia de política exterior, puesto que se había reconocido su posible utilidad en este sentido, si bien al principio gozó de un margen de maniobra relativamente amplio. En los años 1933/1934 se ampliaron las competencias del DAAD con la finalidad de afianzar su posición en el ámbito de la política cultural exterior ${ }^{28}$. Las sedes de Madrid y Barcelona, así como las de París y Londres, quedaron así subordinadas al DAAD. El papel de las filiales en el extranjero resultaba claro: En ellas había de configurarse el trabajo sobre el terreno de acuerdo con las nuevas directrices políticas. Finalmente, a partir de 1937, el Ministerio de Asuntos Exteriores aumentó la centralización de su política cultural. Se dejaba entrever un afán calculado de intervenir en la cúpula de la política cultural alemana en el exterior. Así se produjo una instrumentalización de las actividades de las filiales con el objetivo de integrarlas en la maquinaria política del Ministerio de Asuntos Exteriores como propaganda cultural. No obstante, los acontecimientos subsiguientes afectarían significativamente a la labor de estas entidades: en primer lugar, el trabajo de las sedes se vio muy reducido a causa de la Guerra Civil en España, y después, al estallar la Segunda Guerra Mundial, se dificultó cada vez más la actividad del DAAD en Alemania, hasta que en mayo de 1945 se produjo la disolución de esta organización ${ }^{29}$.

\section{El Instituto GörRes de MadRID: UNA INICIATIVA DE ORIGEN CATÓLICO}

Otra iniciativa de relevancia para el intercambio científicoacadémico entre Alemania y España -si bien con una motivación radicalmente distinta- fue la creación del Instituto Görres de Madrid en el año $1927^{30}$. La Görres-Gesellschaft zur Pflege der Wissenschaft im Katholischen Deutschland se había fundado oficialmente el 25 de enero de 1876 en Coblenza con ocasión del centenario del nacimiento de Joseph von Görres (1776-1848) y en respuesta al Kulturkampf de la era Bismarck, en la que se adoptaron una serie de medidas encaminadas a poner coto a la influencia de la Iglesia católica en Alemania, y muy especialmente en los ámbitos de la ciencia y de la educación ${ }^{31}$. Esta sociedad tenía como objetivo una defensa decidida de los valores de la tradición católica en las ciencias y por un fortalecimiento de la posición de los intelectuales católicos frente a sus colegas protestantes. Originariamente, existía además el proyecto de crear una universidad católica; sin embargo, tal afán no llegaría a cristalizar, a causa del monopolio ejercido por el Estado en materia educativa. De este modo, mediante la intervención de esta sociedad, se intentó ayudar a la ciencia católica a lograr un mayor prestigio e influencia, hacer avanzar la investigación científica desde una perspectiva católica y apoyar a los intelectuales católicos en sus proyectos científicos, como asi se puso de manifiesto en el día de la Iglesia Católica, celebrado en Frankfurt en $1863^{32}$.

Resulta interesante su coincidencia en el tiempo con la creación en Madrid de la Institución Libre de Enseñanza (ILE), que apuntaba justo en dirección contraria: esta institución de enseñanza se habia fundado también en 1876 como alternativa a la universidad española de signo confesional. Al mando se encontraba un grupo de profesores reunidos en torno a Francisco Giner de los Ríos, que tras la Restauración se habían manifestado en contra de la ortodoxia católica y a favor de la libertad religiosa y académica en el ámbito universitario, a raíz de lo cual fueron depuestos de sus cargos. La ILE, inspirada en los postulados del filósofo kantiano Karl Christian Friedrich Krause (1781-1832) y orientada fundamentalmente hacia los principios pedagógicos de Pestalozzi y Fröbel, nació como una iniciativa privada, independiente de Iglesia y Estado, que introdujo en España nuevos métodos de enseñanza y concedía gran valor a la imparcialidad religiosa y política. El hecho de que estas dos iniciativas cristalizaran en España y Alemania de manera simultánea, pero mostraran un desarrollo en direcciones distintas, o mejor dicho, contrarias, es sumamente revelador del contexto histórico en el que se desarrolló posteriormente la ciencia.

La Sociedad Görres entendía por "ciencia católica" "la ciencia de los eruditos católicos que en todas las cuestiones puramente científicas no conocen otras reglas que las de

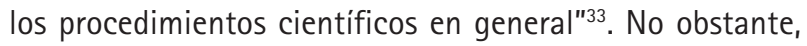
"creía a pies juntillas en el principio de que no hay con- 
tradicción posible entre fe y conocimiento". Con semejante planteamiento, que no permite la existencia de contradicción entre la doctrina de la Iglesia y los resultados de la Ciencia, adquiría singular importancia el alcance internacional que lograra el trabajo. Por esta razón, los institutos existentes en el extranjero tenían especial relevancia, puesto que habian de facilitar el vínculo de la ciencia católica alemana con otros paises: De este modo, en el año 1888 se inauguró en Roma un instituto que se ocupaba fundamentalmente de labores de archivistica, y en 1908 se creó en Jerusalén un Instituto Görres más centrado en tareas arqueológicas ${ }^{34}$.

La iniciativa que desembocó finalmente en la creación de una filial en Madrid se debe a Heinrich Johannes Finke ${ }^{35}$, especialista en Historia de la Iglesia. Ya en el año 1892 había emprendido un viaje a España en busca de fuentes inéditas del Concilio de Constanza, que él localizaba en el Archivo Real de Barcelona. Este viaje había suscitado entre los historiadores alemanes un enorme interés por España. Desde 1901 Finke pergeñaba el plan de crear en España un centro de investigación, para lo cual emprendió nuevos viajes a fin de preparar tal institución. Entre los años 1924 y 1938 Finke estuvo al cargo de la presidencia de la Sociedad Görres y en tal función reforzó, en la medida de sus posibilidades, las relaciones con España. Finalmente, el 22 de septiembre de 1925 la directiva de la Sociedad decidió crear un centro de investigación en España y confió la realización de los preparativos necesarios a una comisión, en la que junto al profesor Finke se encontraban también los profesores Konrad Beyerle y Georg Schreiber ${ }^{36}$.

A tenor de la memoria de la Sociedad Görres, el móvil de su creación fue la esperanza de poder recuperar aún en España -sobre todo en los archivos de Barcelona y Simancas- "valores espirituales incalculables para la propia ciencia", más aún teniendo en cuenta que "los lejanos acontecimientos ocurridos en España están vinculados a nuestra historia". Por lo demás, se trataba de "llevar la ciencia alemana a recónditos países"37. Como en el caso de la creación de las instituciones antes mencionadas, en los prolegómenos del surgimiento del Instituto Görres, se volvió la mirada a Francia, para constatar que, a diferencia de las numerosas, y exitosas, iniciativas en suelo francés, hasta entonces no había existido ninguna organización alemana verdaderamente científica en España, "con excepción de un par de centros de gestión de trabajo con un talante práctico" ${ }^{138}$. Si bien se minimizaba adrede la relevancia de aquellas primeras instituciones, hemos de recordar que tanto el Centro de Intercambio de Madrid como el Centro de Estudios de Barcelona se encontraban por entonces en sus años iniciales. En general se puede decir que el Instituto Görres y el Centro de Intercambio eran bastante complementarios en sus objetivos y mantenian buenas relaciones, que se manifestaron en una estrecha colaboración, por ejemplo en la compra de libros para las bibliotecas ${ }^{39}$.

En un primer momento estaba previsto albergar a la Sociedad Görres en un despacho del Colegio Alemán de Madrid, más tarde un padre espiritual de la comunidad católica alemana había conseguido alquilar el palacete de la Marquesa de Osuna situado en la calle Martín de los Heros número 44 y transformarlo en un centro de la cultura espiritual alemana en Madrid, Hogar Santa María, que ahora albergaría también el Instituto Görres. Por fin, en octubre de 1927 Georg Schreiber, en presencia del embajador alemán en España, Johannes Graf von Welczeck, inauguró el instituto. En los años siguientes, hasta 1933, se fue conformando la biblioteca, especializada en historia cultural medieval de España ${ }^{40}$. Sin embargo, la inauguración había ido precedida de la idea expresada por múltiples partes, de que no debía haber demasiadas instituciones científicas alemanas en España porque eso podía, por un lado, provocar malentendidos entre la ciudadanía y, por otro, podría llegar a trastocar los intereses alemanes. Pero finalmente logró imponerse el argumento de que, como iniciativa católica, el Instituto Görres tenía una orientación conceptual radicalmente distinta a la del Centro de Intercambio creado con anterioridad, puesto que sus intereses se dirigian sobre todo hacia la historia de la Iglesia y la Edad Media.

Por otra parte, el punto fuerte de esta institución fue desde un principio su concepción como centro de investigación; de ahí que en la práctica se dedicara menos al intercambio de personas o publicaciones. Otros objetivos eran la búsqueda de trabajos españoles sobre temas que interesaban al Instituto, para así hacerlos accesibles a la comunidad científica alemana, y el planteamiento de nuevas metas científicas junto con investigadores españoles. Los resultados de tales trabajos se dieron a conocer en las publicaciones de la Sociedad, que aparecían de continuo: la colección "Spanische Forschungen der Görres-Gesellschaft" se inició 
en 1928 y estaba compuesta, por un lado, por los Gesammelte Aufsätze zur Kulturgeschichte Spaniens (desde 1928 hasta 1988 se publicaron 32 volúmenes), y por otro, por las monografías aparecidas desde 1931 hasta hoy día. Además de editar estas publicaciones científicas, la Sociedad Görres organizaba también coloquios científicos hispanoalemanes y financiaba en parte la estancia de investigadores alemanes en España ${ }^{41}$.

Los colaboradores alemanes que durante esta fase inicial habían fijado su residencia en España eran Hugo Obermeier, H. Hüffer y Edmund Schramm. Otros investigadores, por el contrario, seguían su trabajo desde Alemania, realizando viajes de estudio por la Península Ibérica con regularidad. Desde el principio se buscó la estrecha colaboración con España; incluso en la propia junta directiva de la Sociedad Görres había varios científicos españoles y otros muchos destacaban en las publicaciones de la Sociedad, como por ejemplo Z. García Villada, A. Griera, P. Leturia, J. M. Ramos y Loscertales, J. Ruis Serra, Cl. Sánchez Albornoz, M. Torres, F. Valls Taberner y J. Vives ${ }^{42}$.

La suerte que habría de correr posteriormente esta institución estuvo marcada también por la evolución política de ambos paises: por un lado, la proclamación de la República en el año 1931 en España, y, por otro, el nacionalsocialismo en Alemania a partir de 1933. En 1938 fue prohibida en Alemania la Sociedad Görres y, por ser considerada hostil al gobierno del Tercer Reich, su propiedad fue confiscada, teniendo lugar tres años más tarde finalmente su disolución ${ }^{43}$. Durante la Guerra Civil se destruyeron tanto el Instituto de Madrid como su biblioteca. Sin embargo, transcurridos unos pocos años después de la guerra se invirtieron las tornas: en 1949 se reabrió la Sociedad en Alemania y en el año $1951^{44}$ el Instituto madrileño resurgió nuevamente ${ }^{45}$.

\section{Otras iniciativas alemanas en España}

Aunque no cuenten entre las instituciones cientificas stricto sensu, hemos de nombrar aquí los colegios alemanes de España. A ellos acudian en primer término los hijos de ciudadanos alemanes, aunque también niños españoles en los que se despertaba así el interés por la lengua y la cultura alemanas, circunstancia que después facilitaría 0 explicaría una futura posible colaboración con Alemania. Esos colegios surgieron tempranamente en muchas ciudades de España: Barcelona (1894), Madrid (1896), Málaga (1898), Bilbao (1917), Sevilla (1921), y más tarde llegaron otros más en Gijón, San Sebastián, Santander, Vigo, Valencia, Las Palmas, Puerto de la Cruz así como Santa Cruz de Tenerife.

Otra medida encaminada a reforzar la cooperación científico-académica fue la creación en Sevilla de una filial del Instituto Ibero-Americano (IAI), fundado en Hamburgo en 1917. El representante del IAI en la capital hispalense era el profesor Ernst Schäfer, que vivió desde 1924 en Sevilla en la calle Santa Rosa número 2, y que además era delegado de la Asociación de las Universidades Alemanas para España. En octubre de 1920 Schäfer constituyó también en Madrid una delegación española del IAI con sede en el número 9 de la calle Serrano y fundó también finalmente en la primavera de 1921 un círculo local para el intercambio científico hispano-alemán en Palma de Mallorca ${ }^{46}$. Ninguna de estas iniciativas consiguió tener un mayor alcance.

Además, la Academia de Ciencias de Prusia fundó en el año 1927 su "comisión española", que había de promover el contacto con instituciones españolas y tenía la labor de potenciar en España la investigación en el campo de las Humanidades, sobre todo de la Hispanística, de la Historia política y de la Iglesia, de la Historia del Derecho y de la cultura, y de la ciencia de la Antigüedad. En la creación de esta comisión, que a partir del año 1936 se tituló "comisión románica", habían influido tanto los intereses del Ministerio de Cultura de Prusia, favorables a una intensificación de las relaciones hispano-alemanas desde 1917, como los constantes y duraderos esfuerzos de Heinrich Finke y de Georg Schreiber por promover esta colaboración, que culminó en la creación del Instituto Görres ${ }^{47}$.

Dos años más tarde, en 1929, el duque del Infantado y el embajador alemán pusieron en marcha el Comité HispanoAlemán en Madrid con el fin de profundizar las relaciones culturales y científicas entre los dos países, la creación de becas para el intercambio de estudiantes, la organización de conferencias y la fundación de una biblioteca. Poco después esta iniciativa también se llevó a cabo en Barcelona, bajo la presidencia del marqués de Caldas de Monibuy. Al mismo tiempo se crearía una sección local del mismo en 
Sevilla, siendo Hugo Obermaier el encargado de impartir la conferencia inaugural ${ }^{48}$. Este Comité disfrutaba del apoyo tanto del Estado español como del alemán y tenía en cuenta las diferencias y particularidades regionales entre las dos ciudades.

La Notgemeinschaft der deutschen Wissenschaft brindó los medios económicos para la realización de proyectos de investigación en España, si bien éstos partían mayoritariamente de empresarios particulares. En aquellos años surgieron además numerosas sociedades, agrupaciones 0 iniciativas privadas hispano-alemanas, para atraer a investigadores españoles a Alemania.

El gobierno alemán inauguró en mayo de 1941, en plena Segunda Guerra Mundial, el Instituto Alemán de Cultura (Deutsches Wissenschaftliches Institut) de Madrid, bajo la dirección del profesor Theodor Heinermann, un romanista de la Universidad de Münster ${ }^{49}$. Este instituto estaba concebido como herramienta de la política cultural del régimen nazi y seguia las pautas ideológicas de la época. Su edificio se encontraba en el número 35 de la por entonces aún Avenida del Generalísimo y actual Paseo de la Castellana. Poco después, en noviembre de 1942, se creó una sucursal en Barcelona en la Avenida del Generalísimo Franco número 441. Entre los cometidos de este Instituto se hallaban el fomento de la cooperación científica entre ambos paises, del intercambio académico entre las universidades españolas y alemanas, la enseñanza del idioma, la creación de una biblioteca, la concesión de becas, así como el asesoramiento en la búsqueda bibliográfica y la organización de conferencias. El Instituto continuó de esta manera la labor iniciada por el Centro de Intercambio de Madrid, aunque con una orientación ideológica claramente distinta. La biblioteca se basaba en un fundamento de alrededor de 10.000 tomos que quedaban del fondo del Centro ${ }^{50}$. Además, desde principios del año 1942 el Instituto Alemán de Cultura editó de nuevo Boletín Bibliográfico, cuya edición había sido suspendida en julio de 1936, como su revista oficial y bajo la dirección de Antonio Truyol, Theodor Heinemann y Hans Juretschke. Esta institución mantenía una estrecha colaboración tanto con el CSIC, el DAAD y el Instituto lberoamericano como con los romanistas en Alemania ${ }^{51}$. Hasta finales de la Segunda Guerra Mundial pudo continuar con su labor de una manera relativamente tranquila, pero con la capitulación de Alemania su cierre sobrevino inmediatamente.
Por último, hay que mencionar en este contexto la fundación en noviembre de 1943, en plena Segunda Guerra Mundial, del Instituto Arqueológico Alemán (Deutsches Archäologisches Institut) de Madrid ${ }^{52}$, cuyo interés se centró y se sigue centrando en la arqueología de la Península Ibérica y de Marruecos, desde la Prehistoria hasta la Edad Media temprana cristiana y musulmana. Desde comienzos del siglo XIX los arquéologos alemanes habían manifestado un marcado interés por la Península Ibérica y su historia. Tras el trabajo pionero de Emil Hübner ${ }^{53}$, a partir de la finalización de la Primera Guerra Mundial, y en gran medida debido a las investigaciones y excavaciones realizadas por Hugo Obermaier ${ }^{54}$ y Adolf Schulten ${ }^{55}$, las relaciones científicas entre los arqueólogos españoles y alemanes jugaron un importante papel en las relaciones culturales hispanoalemanas, hasta que finalmente fue inaugurada por su primer director Helmut Schlunk ${ }^{56}$ la sección madrileña del Instituto Arqueológico Alemán. Schlunk consiguió alojarla en el entonces llamado Instituto Cultural Alemán, donde podía estar abierta al público. Sin embargo, poco antes del fin de la Segunda Guerra Mundial fueron cerrados todos los departamentos internacionales del DAl, y la recientemente fundada sede madrileña también fue clausurada de manera abrupta ${ }^{57}$.

\section{CONSIDERACIÓN FINAL}

Como se ha podido ver a través de las explicaciones precedentes, a comienzos del siglo XX el interés que desde hacía tiempo existía en Alemania por la Hispanistica se unió al aislamiento científico que, por condicionantes políticos, tuvo lugar tras la Primera Guerra Mundial. En la Europa de los años 20 se daba un consenso generalizado acerca de la importancia de crear instituciones de proyección internacional y destinadas al fomento de la ciencia. Esto hizo proliferar numerosas iniciativas, tanto públicas como privadas, que en los primeros decenios del siglo XX posibilitaron una profundización de las relaciones científicas entre Alemania y España. Este reforzamiento del contacto cultural y académico, aunque a un nivel institucional, se produjo casi simultáneamente en ambos paises y de forma análoga, si bien sobre el trasfondo de unas circunstancias diversas o bien con una orientación conceptual distinta. A pesar de la divergencia en cuanto a intereses de las iniciativas alemanas en relación con la 
JAE - a causa de su retraso científico, esta última estaba interesada en primera instancia en una transferencia de conocimientos de otros paises hacia España, a fin de no perder el tren a escala internacional- se vino a producir, dada la coincidencia en el tiempo de diversos proyectos, una concentración de fuerzas. En cierto modo, puede hablarse de una feliz coincidencia en tiempos difíciles, en los que se buscaba una salida a una situación desastrosa. La dinámica surgida de todo ello impulsó en buena medida el desarrollo de la ciencia en ambos paises y el mutuo intercambio.

Tanto más lamentable resulta por tanto, que fueran las circunstancias políticas las que pocos años más tarde y casi de forma simultánea en ambos países -con el comienzo de la Guerra Civil en España y a continuación el de la Segunda Guerra Mundial- acabaran con tan prometedores planes y proyectos. Si bien es cierto que las relaciones hispano-alemanas no llegaron nunca a romperse, ni siquiera durante la Guerra ${ }^{58}$, y que después de esa difícil época revivieron en parte las instituciones que se habían fundado con anterioridad bajo una nueva forma, no obstante estos sucesos políticos supusieron una enorme pérdida, que se refleja sobre todo a nivel personal; es decir, en su afectación a las personalidades implicadas en ese intercambio científico institucionalizado. Las represalias ordenadas por las dictaduras instauradas, la instrumentalización de la ciencia en provecho de las nuevas ideologías, así como la propia guerra y el exilio que provocó, o incluso la muerte de numerosos investigadores contribuyeron a desbaratar las redes creadas por estos individuos de altas miras para la posterior colaboración, destruyéndose así también el fruto de largos años de denuedos e iniciativas privadas sumamente prometedoras para la cooperación científica hispano-alemana. También los largos años de régimen franquista han contribuido lo suyo a que algunos de los lazos tan provechosos que había entre Alemania y España no pudieran reconstruirse al término de la Segunda Guerra Mundial. En definitiva, se ralentizó así un proceso que ya en el año 1928 se había considerado necesario:

"Más que nunca es tarea de Europa acercar y estrechar los vínculos entre las culturas europeas para reafirmar y profundizar así la peculiar esencia del espíritu europeo"59.

\section{NOTAS}

* Este trabajo se ha realizado en el marco de un proyecto de investigación del Ministerio de Ciencia e Innovación (HAR2010-21333-C03-02). Una primera y abreviada versión de este artículo ha sido publicada recientemente: Rebok, Sandra, "Ciencia alemana en España: los comienzos de la cooperación institucional en los albores del siglo XX", en Sandra Rebok (ed.), Traspasar fronteras: Un siglo de intercambo científico ente España y Alemania. Über Grenzen hinaus: Ein Jahrhundert deutsch-spanische Wissenschaftsbeziehungen. Madrid: CSIC, 2010, pp. 106-137.

1 Sánchez Ron, José Manuel (coord.), 1907-1987. La Junta para la Ampliación de Estudios e Investigaciones Científicas 80 años después, 2 vols.,
Madrid: CSIC, 1988; Puig-Samper, Miguel Ángel (ed.), Tiempos de investigación: JAE-CSIC, cien años de ciencia en España, Madrid: CSIC, 2007.

2 Düwell, Kurt, Deutschlandsauswärtige Kulturpolitik 1918-1932. Grundlinien und Dokumente, Köln/Wien: Böhlau Verlag, 1976, p. 154; Schröder-Gudehus, Brigitte, Deutsche Wissenschaft und Internationale Zusammenarbeit, 1914-1928, Genf: Dumaret un. Golay, 1966, pp. 40 y ss. Véase también: Kerkhoff, Karl, Der Krieg gegen die deutsche Wissenschaft. Eine Zusammenstellung von Kongressberichten und Zeitungsmeldungen, Wittenberg, 1922; Schreiber, Georg, Die Not der deutschen Wissenschaft und der geistigen Arbeiten. Geschehnisse und Gedanken zur Kulturpolitik des Deutschen Reiches, Leipzig, 1923.
Aceptado: 14 de enero de 2011 
3 Marsch, Ulrich, Notgemeinschaft der Deutschen Wissenschaft. Gründung und frühe Geschichte, 1920-1925, Frankfurt a. Main: Peter Lang, 1994; Treue, Wolfgang, Die Notgemeinschaft der Deutschen Wissenschaft und die Deutsche Forschungsgesellschaft, en Rüdiger vom Bruch y Rainer A. Müller (eds.), Formen außerstaatlicher Wissenschaftsförderung im 19. und 20. Jahrhundert. Deutschland im europäischen Vergleich, Stuttgart, 1990.

4 Publicación en versión alemana: "Aufruf spanischer Gelehrter zugunsten der deutschen Wissenschaft". Mitteilungen aus Spanien, año 1, agosto-diciembre 1919, p. 235.

5 Schmidt, Franz, "Anfänge deutscher Kulturpolitik im Auslande", Zeitschrift für Politik, año 3, núm. 3, diciembre de 1956, p. 253.

6 Paul Fridolin Kehr (1860-1944), historiador y diplomático. Desde 1893 Kehr era catédratico en Marburg y a partir de 1895 ejerció este puesto en Gotinga. En 1903 llegó a ser director del Instituto Histórico de Prusia en Roma, entre 1915 y 1929 fue director general de los Archivos Estatales de Prusia, de 1919 a 1935 presidió la Dirección General de la Monumenta Germaniae Historica y hasta el año 1941 ocuparía el puesto de director del Instituto Kaiser Wilhelm para la Historia Alemana, antecesor del Instituto Max-Planck de Gotinga. Desde los inicios del siglo XX promovió la institucionalización de la investigación histórica en Alemania.

7 Vincke, Johannes, "Die spanischen Forschungen der Görresgesellschaft", Historisches Jahrbuch 55, 1935, p. 454.

8 Las siguientes publicaciones ofrecen una visión general sobre las relaciones científicas hispano-alemanas al principio del siglo XX: Hera Martínez,
Jesús de la, La politica cultural de Alemania en España en el período de entreguerras, Madrid: CSIC, 2002; López Sánchez, José María, "Política cultural exterior alemana en España durante la República de Weimar", Cuadernos de historia contemporánea, núm. 25, 2003, pp. 235-253; Förg, Ludwig, "Deutsch-spanische Kulturbeziehungen im wissenschaftlichen Pressearchiv", Gesammelte Aufsätze zur Kulturgeschichte Spaniens. Spanische Forschungen der Görresgesellschaft, tomo 5, primera serie, Münster: Aschendorffsche Verlagsbuchhandlung, 1935; Pöppinghaus, Ernst-Wolfgang, Moralische Eroberungen? Kultur und Politik in den deutsch-spanischen Beziehungen der Jahre 1919 bis 1933, Frankfurt a. M.: Vervuert, 1999. Presas y Puig, Albert, "On a lecture trip to Spain: the scientific relations between Germany and Spain during the Entente boycott (1919-1926)", Annals of Science, 65, núm. 4, 2008, pp. 529546; Schreiber, Georg, "Spanien und Deutschland. Ihre kulturpolitischen Beziehungen", en K. Beyerle, Heinrich Finke y Georg Schreiber (eds.), Gesammelte Aufsätze zur Kulturgeschichte Spaniens. Spanische Forschungen der Görresgesellschaft, tomo I, primer serie, Münster/Westfalia: Aschendorffsche Verlagsbuchhandlung, 1928, pp. 1-92.

9 Schairer, Reinhold, Die Studenten im Internationalen Kulturleben, Münster/Westfalia: Aschendorffsche Verlagsbuchhandlung, 1927, p. 91.

10 Praesent, Hans, "Die Deutsche Wissenschaftliche Vermittlungsstelle in Barcelona", Minerva-Zeitschrift, n. 1, cuaderno 3, 1924/25, pp. 93-95.

11 Schreiber, 1928, p. 55.

12 PAAA, R 64481. Esbozo de programa con fecha de 6 de febrero de 1923.

13 Pöppinghaus, 1999, p. 285.
14 Schreiber, 1928, p. 38.

15 Georg Schreiber (1882-1963). Politico e historiador de la Iglesia, nació el 5 de enero de 1882 en Rüdershausen (cerca de Duderstadt) en el seno de una familia de guardabosques. Después de estudiar Teología, Historia y Ciencias Jurídicas en Münster y Berlín, fue ordenado sacerdote en el año 1905. En 1909 se doctoró en Filosofía en Berlín y en 1913 en Teología por la Universidad de Friburgo. En 1917 obtuvo en Münster una cátedra de Historia medieval y moderna de la Iglesia y ciencias diaconales. Entre 1920 y 1933 fue diputado del Reichstag por el Centro y durante la República de Weimar marcó en buena medida la política cultural y científica católica. Además fundó en 1927 el Deutsches Institut für Auslandskunde en Münster y en marzo de 1933 el Institut für Volkskunde. Tras la subida al poder de los nacionalsocialistas fue sometido a represalias, por lo que en 1936 solicitó su jubilación anticipada. A comienzos de 1938 fueron confiscados por la Gestapo los dos centros de investigación de Münster junto con sus bibliotecas y se inició contra él un proceso penal de sesgo político. Como consecuencia de la ola de detenciones posteriores al 20 de julio de 1944, pasó a la clandestinidad y encontró refugio en el convento benedictino de Ottobeuren hasta la liberación de los aliados. En la inmediata posguerra fue el primer rector de la Universidad de Münster, colaborando con numerosas organizaciones científicas en la reconstrucción de la ciencia alemana. Desde 1951 hasta su muerte, acaecida el 24 de febrero de 1963 en Münster, fue miembro científico del Max-PlanckInstituts für ausländisches Recht und Völkerrecht de Heidelberg. 
16 PAAA, R 64478. Kult VI B. Kunst und Wissenschaft, núm. 282, informe de Harnack a Simons con fecha de 2 de julio de 1920. Véase también Hera Martínez, 2002, pp. 76-79; Pöppinghaus, 1999, pp. 261-280.

17 Moldenhauer, Gerhard, "Aus der Tätigkeit der Arbeitsstelle für deutschspanische Wissenschaftsbeziehungen in Madrid", Iberoamerikanisches Archiv, año 3, núm. 1, marzo de 1929, p. 11.

18 Se trata del mismo edificio que alberga hoy dia, si bien con una dirección distinta, el Goethe-Institut de Madrid.

19 Gerhard Moldenhauer (1900-1980). Filólogo, nació el 19 de enero de 1900 en Unterpeissen (cerca de Bernburg). Su padre era pastor protestante. Entre 1918 y 1922 cursó estudios de Teología, Filología Románica e Historia en Halle y Hamburgo. Se doctoró con el profesor Karl Voretzsch. Desde 1922 hasta 1925 emprendió diversos viajes de estudios a España, Sudamérica y Portugal. En 1925 fue nombrado director del nuevo Centro de Relaciones Científicas Hispanoalemanas de Madrid (hasta 1929). En 1926 se habilitó como catedrático de Filología Románica en Halle, pero mantuvo su responsabilidad en el Centro madrileño, circunstancia por la que no acometió su labor docente. Tres años después se habilitó en la Universidad de Bonn y entre 1938 y 1945 impartió su docencia en la Universidad de Viena. Sin embargo, hasta 1945 Moldenhauer fue revelándose como dogmático nacionalista, fue miembro del NSDAP y de la división naval de las SA. A partir de 1933 desempeñó un papel activo en la asociación de profesores nacionalsocialistas, así como en otras agrupaciones. Al término de la Guerra fue despedido, si bien pudo continuar su actividad docente en Argentina. Alli obtuvo contratos temporales en 1949 y 1957 en Buenos Aires, y en 1950 en Rosario.

20 Moldenhauer, 1929, p. 2.

21 Ya en el año 1927, por ejemplo, se dio respuesta por escrito a 5.000 consultas.

22 Adams, Alfons, "Deutsche wissenschaftliche Institute in Spanien", Hochschule und Ausland, julio 1931, cuaderno 7, p. 10.

23 Una relación detallada de los temas de las conferencias se encuentra en Moldenhauer, 1929, pp. 5-7.

24 El 1 de octubre de 1925 trasladó su sede a Berlín.

25 Esta iniciativa precursora fue inscrita en el registro oficial en 1923 por Alfred Weber, Arnold Bergstraesser, Carlo Joachim Friedrich y Eberhard Gothein en Heidelberg con el nombre de "Deutsche Vereinigung für staatswissenschaftlichen Studentenaustausch e.V.".

26 Para una descripción más precisa de esta primeras organizaciones véase: Laitenberger, Volkhardt, Akademischer Austausch und auswärtige Kulturpolitik. Der Deutsche Akademische Austauschdienst (DAAD) 1923-1945, Göttingen: Musterschmidt, 1976, pp. 16-33.

27 DAAD, Informe de actividad 1931/32, citado en ibidem, 1976, pp. 46-47. En el número 7 de esta misma revista, de julio 1931, ofrecen por ejemplo detallados consejos sobre los estudios en España ("Ratschläge für das Studium in Spanien", pp. 25-28).

28 Laitenberger, 1976, pp. 60-72.

29 Finalmente el 1 de enero de 1950 se reinició oficialmente la actividad del DAAD en Alemania y desde el año 2004 está de nuevo representado con una oficina en España (Barcelona).
30 Hernández Ariza, Javier, "Institutos Hispano-alemanes en Madrid: La Görresgesellschaft y el Deutsches Archäologisches Institut", en Miguel Ángel Vega Cernuda (cood.), Relaciones hispano-alemanas: Prejuicios $y$ estereotipos, encuentros y desencuentros: un balance, Alicante: Universidad de Alicante, 2008, pp. 246252; Vincke, 1935, p. 454; Schreiber, 1928, p. 55.

31 Aldea, Quintín, "Una Asociación ejemplar de intelectuales: La Sociedad Görres", Arbor, núm. 338, febrero de 1974 , p. 259.

32 Ibidem, p. 260.

33 Informe anual de 1897, citado en: Finke, Heinrich, "Internationale Wissenschaftsbeziehungen der Görresgesellschaft", en Kronen, H. y Steffes J.P. (eds.), Volkstum und Kulturpolitik, Köln: Gilde-Verlag, 1932, p. 132.

34 Más información en: ibidem, pp. 125-142.

35 Heinrich Johannes Finke (18551938). Historiador de la Iglesia y medievalista, nacido el 13 de junio de 1855 en Knechting (Westfalia) en el seno de una familia modesta. Hubo de financiarse por sus propios medios sus estudios de Filosofía e Historia en Münster y más tarde en Tubinga. Se doctoró en el año 1879 en Tubinga y en 1887 se habilitó en la universidad de Münster. Seguidamente obtuvo una plaza como docente privado de Historia de la Iglesia en Münster y Friburgo, y en 1892 realizó su primer viaje de investigación a España. Finalmente fue llamado en 1898 a Friburgo para tomar el relevo de Aloys Schulte y en 1899 obtiene el puesto de catedrático de Historia en la "cátedra confesional católica". A partir de 1920 fue vicepresidente de la Sociedad Görres y entre 1924 y 1938 presidente. Fue nombrado miembro 
honorífico de la Real Academia de Historia de Madrid, así como de la Real Academia de Buenas Letras de Barcelona y fue investido doctor honoris causa por las universidades de Barcelona, Salamanca, Valladolid y Madrid. Finke estuvo en continuo diálogo con eruditos españoles, dirigió a más de 30 doctorandos españoles y abogó a lo largo de toda su vida por la cooperación científica hispano-alemana. Cuando murió en Friburgo el 19 de diciembre de 1938 , era considerado como uno de los historiadores de la Iglesia más célebres y de mayor renombre, que gozó de un gran prestigio sobre todo en España.

36 Vincke, Johannes, "Wilhelm Neuss und die Anfänge der 'Spanischen Forschungen'", Gesammelte Aufsätze zur Kulturgeschichte Spaniens. Spanische Forschungen der Görresgesellschaft, vol. 18, Münster: Aschendorffsche Verlagsbuchhandlung, 1961, p. 327.

37 Véase memoria de la Sociedad Görres del 27 de julio de 1925, publicada en: Vincke, 1961, pp. 328-329.

38 Véase memoria de enero de 1926 publicada en ibidem, p. 334.

39 Adams, 1931, p. 12.

40 Vincke, 1935, p. 459.

41 En los pocos años transcurridos entre su fundación en 1927 y el estallido de la Guerra Civil en 1936, 30 investigadores alemanes pudieron disfrutar de tales ayudas.

42 Vincke, 1935, p. 463.

43 Engels, Odilo, "La historia de la Sociedad Görres y su contribución a la cultura española y la ciudad de Madrid", en Javier Prades (ed.), La esperanza en el mundo globalizado, Madrid: Facultad de Teología "San Damaso", p. 132.

44 La inauguración oficial del instituto en su nueva sede tuvo lugar el 26 de agosto de 1955.
45 En 1953 se estableció inicialmente en el edificio del Consejo Superior de Investigaciones Cientificas de la calle Serrano 117, primero bajo la dirección de Johannes Vincke y siendo posteriormente Hans Juretschke quien, con la colaboración del Padre Quintín Aldea, asumiria esta función. Desde 1986 el Instituto Görres, junto con su biblioteca, se encuentra en el edificio del Seminario Conciliar de la calle San Buenaventura en Madrid. Desde 1997 hasta 2002 la Fundación Deutsche Stiftung de Madrid se hizo cargo de la biblioteca e inmediatamente se entregaron sus fondos a la Facultad de Teología "San Dámaso", que se encuentra en el edificio aledaño al Seminario. Hoy en día la biblioteca de Humanidades, considerablemente ampliada, cuenta con más de 30.000 volúmenes y revistas del ámbito de la literatura, filosofía, teología, historia y geografía (entre los que cabe destacar una extensa colección especial con libros antiguos de viajes).

46 Carta del Consulado General de Barcelona a la Embajada de Madrid fechada el 10 de junio de 1921. PAAA, KV 2559/21.

47 Boehm, Laetitia, "Langzeitvorhaben als Akademieaufgabe. Geschichtswissenschaften in Berlin und in München", en Wolfram Fischer, Rainer Hohlfeld y Peter Nötzoldt (eds.), Die Preussische Akademie der Wissenschaften zu Berlin 1914-1945, Berlin: Akademie Verlag, pp. 407 ss.

48 Förg, 1935, p. 425.

49 Truyol Serra, Antonio, "El nuevo Instituto Alemán de Cultura", Investigación y Progreso, XII, núm. 6, junio de 1941, pp. 225-230; Hausmann, Frank-Rutger, "Auch im Krieg schweigen die Musen nicht". Die Deutschen Wissenschaftlichen Institute im
Zweiten Weltkrieg, Göttingen: Vandenhoeck und Ruprecht, 2001, pp. 211-237; "El Instituto Alemán de Cultura de Madrid, lazo que une y coordina las relaciones intelectuales entre Alemania y España", $A B C, 23$ de noviembre de 1941, p. 16.

50 Hausmann, 2001, p. 225.

51 Ibidem, p. 214.

52 Marzoli, Dirce, Maier Jorge y Schattner, Thomas (eds.), Historia del Instituto Arqueológico Alemán de Madrid, Madrid, 2010 (en prensa).

53 Emil Hübner (1834-1901). Emil Hübner nació en Düsseldorf el 7 de julio de 1834. Estudió el bachillerato en Dresde, para a continuación iniciar sus estudios en la Universidad de Berlín, donde se doctoró en 1854 bajo la dirección de Friedrich Ritschl (1806-1876). Completó su formación en Italia y Sicilia. En 1858 fue invitado por Theodor Mommsen (18171903) para colaborar en el Corpus Inscriptionum Latinarum y redactar el volumen correspondiente a Hispania. Con este motivo viajó por España, país que desde entonces y hasta el final de sus días se convirtió en su principal campo de investigación. También le fue encargado el volumen sobre Britannia. Fue profesor de Filología Clásica en la Universidad de Berlín desde 1863 y miembro de la Real Academia de Ciencias de Berlín. Falleció en esa misma ciudad el 21 de febrero de 1901.

54 Hugo Obermaier (1877-1946). Paleontólogo alemán, en 1910 llega a España para excavar la cueva de El Castillo en Cantabria, en lo que sería la primera misión del Institut de Paléontologie Humaine de Paris. El estallido de la Primera Guerra Mundial le impide regresar a Francia por su condición de ciudadano alemán. Se inicia entonces la etapa 
netamente española de Obermaier: por mediación del Conde de la Vega del Sella, se integra en la Comisión de Investigaciones Paleontológicas y Prehistóricas, trasladándose luego al Museo Nacional de Ciencias Naturales de Madrid. En 1922 se crea para él la cátedra de "Historia Primitiva del Hombre" en la Universidad Central de Madrid y se le otorga una plaza en la Academia de la Historia. Tras el estallido de la Guerra Civil aceptará una cátedra en la Universidad de Friburgo (Suiza), donde permanecerá hasta su muerte acaecida en 1946.

55 Adolf Schulten (1870-1960). Historiador, estudió en las universidades de Gotinga, Bonn y Berlín. Fue profesor en Gotinga desde 1896, y en Erlangen desde 1907, donde será nombrado catedrático en 1909. En 1899 y en 1902 viaja a España, interesándose por las ruinas de Numancia. Entre 1905 y 1914 llevó a cabo diversas campañas arqueológicas en esta ciudad celtíbera. La Primera Guerra Mundial interrumpió la actividad arqueológica de Schulten en España, aunque, terminado el conflicto, volvió inmediatamente en 1919 para estudiar, gracias a iniciativas privadas y al Instituto de Estudios Catalanes, la costa española mediterránea, lo que le llevaría a buscar la mítica ciudad de Tartessos. Proyectó la publicación de los Fontes Hispaniae Antiquae (FHA), que, en varios volúmenes, debian recoger todos los textos antiguos y altomedievales referentes a Hispania. La Real Academia de la Historia le nombró Miembro Correspondiente en 1905 y en 1907 el Rey le concedió la "Encomienda de número" de la Orden de Alfonso XII. En 1940 recibió de Franco la Gran Cruz de la Orden de Alfonso X el Sabio Y, de la Universidad de Barcelona, el doctorado honoris causa.

56 Helmuth Schlunk (1906-1982). Historiador del Arte de la Alta Edad Media, en 1928 realizó su primer viaje a España, becado por el Estado alemán, para estudiar las pinturas murales asturianas. En 1942 fue encargado de crear el Instituto Arqueológico Alemán de Madrid, inaugurado en 1943 y clausurado por las autoridades aliadas en 1945. Durante los difíciles años subsiguientes, Schlunk fue nombrado profesor de la Universidad de Valencia y del Instituto Diego Velázquez del Consejo Superior de Investigaciones Científicas. Asi pudo continuar su labor investigadora hasta la reapertura del Instituto Arqueológico Alemán en 1953, del que sería nombrado director hasta su jubilación, en 1971. Su labor científica se centrará entonces en la excavación del mausoleo de Centcelles, así como en las campañas realizadas, junto al profesor Hauschild, en el gran mausoleo de Puebla Nueva (Toledo) y en la iglesia de Marialba, cerca de León. En 1959 creó la revista
Madrider Mitteilungen. Fue investido doctor honoris causa por las universidades de Sevilla, Oviedo y Salamanca y nombrado miembro numerario y correspondiente de diversas academias e instituciones científicas españolas y extranjeras.

57 Su reapertura se llevó a cabo en marzo de 1954, nuevamente bajo la dirección de Schlunk.

58 Para más información sobre este período, véase: Marquina Barrio, Antonio, "La política exterior de España en Alemania durante la Segunda Guerra Mundial", en Bernecker, Walther (ed.), España y Alemania en la Edad Contemporánea, Frankfurt a.M.: Vervuert, 1992, pp. 173-195; Sanz Díaz, Carlos, "El papel de la política cultural en las relaciones hispano-alemanas, 1949-1966", Ayer, núm. 69, 2008, pp. 155-185; Janué i Miret, Marició, "La cultura como instrumento de la influencia alemana en España: la Sociedad Germano-Española de Berlín (1930-1945)", en Janué i Miret, Marició (ed.), España y Alemania: Nuevas investigaciones sobre la historia de las relaciones culturales en el siglo XX, Ayer 69 (dossier), 2008; Ruiz Escudero, Inés, "Las relaciones entre la República Federal de Alemania y el Régimen Franquista tras la Segunda Guerra Mundial a través de la prensa española (1945-1958)", HOAL, núm. 12, invierno 2007, pp. 133-141.

59 Schreiber, 1928, p. 62. 